\title{
Current clinical applications of platelet-rich plasma in various gynecological disorders: An appraisal of theory and practice
}

\author{
Ayman Shehata Dawood, Hesham Abdelaziz Salem \\ Department of Obstetrics and Gynecology, Tanta University, Tanta, Egypt
}

The purpose of this paper is to review the current clinical uses of platelet-rich plasma (PRP) in the field of gynecology. All relevant articles published from January 2000 to December 2017 were reviewed and analyzed. The articles on PRP in the field of gynecology were mainly case series, pilot studies, or case reports. PRP is currently considered a new therapeutic modality for some disorders that are refractory to conventional drugs.

Keywords: Endometrium; Gynecology; Infertility; Platelet-rich plasma

\section{Introduction}

Platelet-rich plasma (PRP) is becoming more popular as a nonoperative treatment option for a broad spectrum of medical disorders. PRP is widely used in orthopedic and sports medicine to relieve pain through the natural promotion of healing in musculoskeletal diseases such as tendonitis, arthritis, ligament sprains, and tears. In particular, PRP injections have been used for athletic injuries, resulting in exceptional healing, a rapid return to regular activities, and complete pain relief [1].

Autologous PRP is derived from an individual's whole blood then centrifuged to remove red blood cells. The remaining plasma has a 5to 10-fold higher concentration of growth factors than whole blood. These growth factors have been found to promote natural healing responses by researchers across multiple specialties, such as dentist-

Received: Feb 10, 2018 · Revised: May 3, 2018 · Accepted: May 25, 2018

Corresponding author: Ayman Shehata Dawood

Department of Obstetrics and Gynecology, Tanta University, Tanta 31111, Egypt

Tel: +20-1225880071 Fax:+20-0403354567

E-mail:ayman.dawood@med.tanta.edu.eg

This is an Open Access article distributed under the terms of the Creative Commons Attribution Non-Commercial License (http://creativecommons.org/licenses/by-nc/4.0/) which permits unrestricted non-commercial use, distribution, and reproduction in any medium, provided the original work is properly cited. ry, dermatology, urology, and gynecology [2,3].

The theory underlying this treatment modality was derived from natural healing processes, as the body's first response to tissue injury is to deliver platelets to the injured area. Platelets promote healing and attract stem cells to the site of the injury. Moving from basic science to clinical practice, PRP injections have been applied to diseased ligaments, tendons, and joints, with superb outcomes in terms of repair [4]. The objective of this study was to review studies addressing the use of PRP in the field of gynecology, in light of the limited experiences of the use of PRP to treat gynecological disorders.

\section{Methods}

A literature search was done using PubMed, Google Scholar, Clinical Key, and Medline to identify studies investigating the uses of PRP in gynecology. The search period was from January 1, 2000 to December 31, 2017. The search words were "platelet-rich plasma," "implementations," "uses," "endometrium," "infertility," and "gynecology." The studies found were case series, case reports, letters, and pilot studies with a small sample size. No randomized clinical trials with a suitable number of patients were found in the search results. 


\section{The science of PRP}

\section{1) PRP preparation}

The preparation of PRP is an outpatient procedure that involves a blood draw, preparation of the PRP, and the injection of PRP into the diseased area. Multiple methods have been developed for PRP preparation, with variation in the speed and timing of centrifugation $[5,6]$. The following steps present a representative method of preparing PRP: (1) venous blood (15-50 $\mathrm{mL}$ ) is drawn from the patient's arm in anticoagulant-containing tubes; (2) the recommended temperature during processing is $21^{\circ} \mathrm{C}-24^{\circ} \mathrm{C}$ to prevent platelet activation during centrifugation of the blood; (3) the blood is centrifuged at 1,200 rpm for 12 minutes; (4) the blood separates into three layers: an upper layer that contains platelets and white blood cells, an intermediate thin layer (the buffy coat) that is rich in white blood cells, and a bottom layer that contains red blood cells; (5) the upper and intermediate buffy layers are transferred to an empty sterile tube. The plasma is centrifuged again at 3,300 rpm for 7 minutes to help with the formation of soft pellets (erythrocytes and platelets) at the bottom of the tube; (6) the upper two-thirds of the plasma is discarded because it is platelet-poor plasma; (7) pellets are homogenized in the lower third ( $5 \mathrm{~mL}$ ) of the plasma to create the PRP; (8) the PRP is now ready for injection. Approximately $30 \mathrm{~mL}$ of venous blood yields $3-5 \mathrm{~mL}$ of PRP; (9) the affected area is disinfected before the PRP injection; (10) providing assurance to the patient and discussing the procedure make the injection easier and less painful; (11) PRP stimulates a series of biological responses, and the injection site may become swollen and painful for roughly 3 days.

\section{2) Types of PRP preparations}

PRP preparations can be classified according to the preparation method, the content of the sample, and the proposed application. Preparations vary in terms of centrifugation speed, centrifugation time, and anticoagulant use, while the content varies depending on the predominant constituent (e.g., platelets, leukocytes, or growth factors) [7].

After centrifugation of whole blood, four types of preparations can be obtained, as shown in Table 1. This classification was proposed by Dohan Ehrenfest et al. [8], and is based on cell content and fibrin density. This classification was recommended by a multi-disciplinary consensus committee [9].

Table 1. Platelet-containing preparations, as classified by Dohan Ehrenfest et al. [8]

\begin{tabular}{lccc}
\hline Preparation & Acronym & Leukocytes & Fibrin density \\
\hline Pure platelet-rich plasma & P-PRP & Poor & Low \\
Leukocyte- and platelet-rich plasma & L-PRP & Rich & Low \\
Pure platelet-rich fibrin & P-PRF & Poor & High \\
Leukocyte- and platelet-rich fibrin & L-PRF & Rich & High \\
\hline
\end{tabular}

Another classification of PRP preparations was proposed by Mishra et al. [10] based on the presence or absence of white blood cells, their activation status, and platelet concentration. A newer classification of PRP preparations was introduced by Magalon et al. [11] and called the DEPA (dose of injected platelets, efficiency of production, purity of the PRP, activation of the PRP) classification, as shown in Table 2.

\section{3) PRP composition and activation}

Platelets contain high concentrations of cytokines and growth factors stored within a-granules. These growth factors include plateletderived growth factor, insulin-like growth factor, vascular endothelial growth factor, platelet-derived angiogenic factor, transforming growth factor beta, fibroblast growth factor, epidermal growth factor, connective tissue growth factor, and interleukin-8. In addition to growth factors, platelets contain other substances, such as fibronectin, vitronectin, and sphingosine 1-phosphate, that initiate wound healing [12,13].

Platelet activation triggers the release of these growth factors by a variety of substances or stimuli such as thrombin, calcium chloride, and collagen. Each method influences both the physical form of PRP and the amount of growth factors released, as well as the kinetics of release. No evidence has been found regarding the ideal concentration of activator required to trigger the optimal release of growth factors during the activation process of PRP, and different concentrations may therefore lead to different results [12].

\section{4) Proposed mechanisms of action of PRP}

The mechanisms of PRP have not been completely elucidated, but laboratory studies have shown that the high concentration of growth factors in PRP can potentially speed up the healing process [13] Growth factors promote wound healing by initiating the following

Table 2. DEPA classification of PRP preparations

\begin{tabular}{|c|c|c|}
\hline DEPA classification & Subgroup & Description \\
\hline $\begin{array}{l}\text { Dose of injected } \\
\text { platelets }\end{array}$ & $\begin{array}{l}\text { Very high } \\
\text { High } \\
\text { Medium } \\
\text { Low }\end{array}$ & $\begin{array}{l}>5 \text { Billion injected platelets } \\
3-5 \text { Billion injected platelets } \\
\text { 1-3 Billion injected platelets } \\
<1 \text { Billion injected platelets }\end{array}$ \\
\hline $\begin{array}{l}\text { Efficiency of } \\
\text { production }\end{array}$ & $\begin{array}{l}\text { High device efficiency } \\
\text { Medium device } \\
\text { efficiency } \\
\text { Low device efficiency } \\
\text { Poor device efficiency }\end{array}$ & $\begin{array}{l}\text { Recovery rate in platelets }>90 \% \\
\text { Recovery rate in platelets } 70 \%-90 \% \\
\text { Recovery rate in platelets } 30 \%-70 \% \\
\text { Recovery rate in platelets }<30 \%\end{array}$ \\
\hline Purity of the PRP & $\begin{array}{l}\text { Very pure PRP } \\
\text { Pure PRP } \\
\text { Heterogeneous PRP } \\
\text { Whole-blood PRP }\end{array}$ & $\begin{array}{l}\text { Platelets in the PRP }>90 \% \\
\text { Platelets in the PRP } 70 \%-90 \% \\
\text { Platelets in the PRP } 30 \%-70 \% \\
\text { Platelets in the PRP }<30 \%\end{array}$ \\
\hline Activation process & $\begin{array}{l}\text { Autologous thrombin } \\
\text { Calcium chloride }\end{array}$ & - \\
\hline
\end{tabular}

Modified from Magalon et al. BMJ Open Sport Exerc Med 2016;2:e000060 [11]. DEPA, dose of injected platelets, efficiency of production, purity of the PRP, activation of the PRP; PRP, platelet-rich plasma. 
stages: tissue necrosis resolution, chemotaxis, cell regeneration, cell proliferation and migration, extracellular matrix synthesis, remodeling, angiogenesis, and epithelialization [14].

PRP is superior to recombinant human growth factor because platelet activation leads to the release of multiple growth factors and differentiation factors. Recently, the fibrin framework present over platelets was found to support the regenerative matrix, leading to the rapid establishment of the proper morphological and molecular configuration for wound healing [15].

\section{Applications of PRP in various gynecological disorders}

Tissue repair begins with clot formation and is followed by platelet degranulation with the release of platelet growth factors. These are necessary and well-regulated processes to achieve wound healing. PRP preparations are used in gynecology for various diseases based on its known mechanisms, which involve the wound healing process and the initiation of inflammatory reactions [2].

\section{1) PRP in skin lesions and wound healing}

Due to the ability of PRP to promote angiogenesis and wound healing, it is widely used in dermatology for purposes including the treatment of ulcers, scars, and alopecia. With this in mind, PRP was tested by Tehranian et al. [16] in wound healing in high-risk women undergoing cesarean sections. They applied PRP in 70 patients and compared them to 71 control cases without PRP application. The inclusion criteria were a body mass index (BMI) $>25 \mathrm{~kg} / \mathrm{m}^{2}$, prior cesarean section, diabetes, twin pregnancy, use of corticosteroid medication, and anemia. They found a greater reduction in the redness, edema, ecchymosis, discharge, approximation score than in the control group ( $85.5 \%$ reduction in the PRP group vs. $72 \%$ in the control group) ( $p<0.001)$. They concluded that PRP was an effective therapeutic approach for wound healing, and faster wound healing can be expected when PRP is used due to the presence of more platelets and growth factors.

Another study was conducted of 55 patients undergoing major gynecological surgery, in whom PRP was directly applied to the surgical site. The authors found that autologous platelet grafts applied in gynecological surgery were effective for pain reduction and were not associated with any adverse effects [17].

\section{2) PRP in cervical ectopy}

Hua et al. [18] conducted a randomized clinical study to compare the effectiveness of autologous PRP application to that of laser treatment for benign cervical ectopy. They applied PRP twice on the area of cervical erosion with a 1-week interval in 60 patients, while laser treatment was used in the other 60 patients. They found that the complete cure rate was $93.7 \%$ in the PRP group and $92.4 \%$ in the laser group ( $p>0.05$ ). The mean time to re-epithelialization was significantly shorter in the PRP group $(p<0.01)$. The rate of adverse treatment effects (i.e., vaginal discharge or vaginal bleeding) was much lower in the PRP group than in the laser group $(p<0.01)$. They concluded that autologous PRP application appeared promising for the treatment of cervical ectopy in symptomatic women, as it yielded a shorter tissue healing time and milder adverse effects than laser treatment.

\section{3) PRP in vulvar dystrophy}

PRP has been tried in many dermatological and autoimmune conditions nonresponsive to corticosteroids, such as lichen sclerosus (LS) and eczema. LS affects the vulva and causes extensive scarring, with progressive loss of the labia minora, sealing of the clitoral hood, and burying of the clitoris. LS also causes progressive pruritus, dyspareunia, and genital bleeding. It has a considerable impact on the quality of life of affected patients by disturbing physical activity, sexual pleasure, and causing emotional and psychological problems [19].

This condition is treated by topical and systemic corticosteroids. Application of PRP in cases of LS resistant to steroid therapy was tried by Behnia-Willison et al. [20] in 28 patients with LS. They injected PRP into the vulva in a fanning pattern. Patients received three PRP treatments 4 to 6 weeks apart and again at 12 months. Nearly all patients exhibited clinical improvements in the size of their lesions, and in $28.6 \%$ of the patients, the lesions disappeared completely after PRP treatment. Minimal pain and no complications were reported. They concluded that PRP injections could therefore be considered an effective therapy for $L S$.

\section{4) PRP in reconstructive surgery for vulvar cancer}

Morelli et al. [21] conducted a retrospective study of patients who underwent surgery for vulvar cancer (radical vulvectomy). The aim of their study was to evaluate the efficacy of platelet gel application in women after radical surgery. They divided patients into two groups: group $A(n=10)$, who had platelet gel placed on the vaginal breach during reconstructive surgery, and group $B(n=15)$, who underwent only surgical treatment. They found significantly lower rates of wound infection $(p=0.032)$, necrosis of vaginal wounds $(p=0.096)$, and wound breakdown $(p=0.048)$ in group A than in group B. They also found a reduction in the postoperative fever rate, a shorter hospital stay, and faster wound healing in group A, which received PRP gel treatment. They concluded that platelet gel application before vulvar reconstruction represented an effective strategy for preventing wound breakdown after surgery to treat locally advanced vulvar cancer.

\section{5) PRP in urogenital disorders}

(1) PRP in genital fistulae

Genital fistulae are treated by many modalities, as listed by Bodner- 
Adler et al. [22] in a systemic review that assessed conservative and surgical treatments. They found that small fistulae could be treated conservatively with various therapies, including PRP, with success rates ranging from $67 \%$ to $100 \%$.

PRP has been tried in the treatment of vesicovaginal fistulae as a novel, minimally invasive approach for the closure of genital fistulae. Shirvan et al. [23], in a series of 12 patients, injected PRP around the fistula into the tissue, and platelet-rich fibrin (PRF) glue was interpositioned in the tract. They followed the cases for 6 months and found that 11 patients were clinically cured, with normal findings on transvaginal physical examinations and cystography. They concluded that autologous PRP injection and PRF glue interposition offered a safe, effective, and novel minimally invasive approach for the treatment of vesicovaginal fistulae that obviated the need for open surgery.

Mongardini et al. [24] presented a case of a complicated iatrogenic low rectovaginal fistula treated by interposition of buccal mucosa and apposition of PRP. Similarly, Gottgens et al. [25] performed PRP injections into the fistula tract after a mucosal advancement flap in 10 patients with Crohn disease-related high perianal fistulae. They found that healing of the fistula took place in $70 \%$ of cases ( $95 \%$ confidence interval, 33\%-89\%) at 1 year. One patient (10\%) experienced recurrence, and in two patients (20\%), the fistula persisted after treatment. They concluded that this procedure was moderately successful in Crohn disease fistulae, with a success rate of 70\%, and suggested that further studies were needed.

\section{(2) PRP in genital prolapse and urinary incontinence}

Both absorbable and nonabsorbable vaginal implants used in pelvic floor reconstructive procedures have numerous serious adverse effects. PRF is a mixture of platelets, leukocytes, cytokines, and circulating stem cells that is optimal for stimulating fibroblast migration and proliferation. This mixture causes rapid remodeling and connective tissue growth after vaginal surgery [26].

Gorlero et al. [26] conducted a prospective observational study of 10 consecutive women requiring surgery for prolapse recurrence (stage II or higher). They operated on the cases and performed PRF injections. The success rate was $80 \%$ with complete symptom relief. Sexual activity increased by $20 \%$ without dyspareunia. They concluded that the use of PRF for site-specific prolapse repair was associated with good functional outcomes. Furthermore, Medel et al. [27] found that the attachment of fibroblasts to vaginal meshes significantly increased after coating the meshes with PRP in vitro.

Recently Chrysanthopoulou et al. [28] summarized the existing evidence from animal experimental and clinical studies regarding the potential role of PRP in treating genital prolapse. They concluded that PRP restores the anatomy and function of pelvic ligaments, but no evidence has yet emerged to support or oppose PRP use in wom- en suffering from genital prolapse. Similarly, Nikolopoulos et al. [29] summarized studies advocating the use of PRP in urinary incontinence resulting from damage to the pubourethral ligament. They found that PRP helps to regulate tissue reconstruction and the restoration of pubourethral ligament strength, but the studies did not provide sufficient evidence to justify its use.

In contrast, another study was conducted to evaluate whether autologous platelet gel application during anterior colporrhaphy increased the collagen content of the pubocervical fascia, resulting in more durable repair. The researchers applied autologous platelet gel to the surgical site during anterior colporrhaphy in nine patients. Biopsy specimens from the anterior vaginal wall during surgery and 3 months postoperatively were collected. They found that no significant increase had occurred in collagen content at 3 months after the operation, and concluded that autologous platelet gel did not increase collagen levels or improve the durability of the repair [30].

\section{PRP in reproductive medicine 1) $P R P$ in premature ovarian failure}

Premature ovarian failure (POF) refers to loss of normal function of the ovaries before age 40 , accompanied by the loss of fertility. A team of researchers at Harvard University injected murine ovaries with growth factors, and mature eggs appeared to develop from ovarian stem cells. They stated that the introduction of isolated growth factor-bearing platelets directly into the ovaries might trigger a resurgence in oocyte production [31].

PRP therapy is investigated in women with POF, infertile women more than 35 years of age, and women with low ovarian reserve. Treatment with PRP is referred to as ovarian rejuvenation; in this procedure, PRP is injected into the ovary under ultrasound guidance, similarly to ovum retrieval in in vitro fertilization (IVF). This modality of treatment is still being investigated in trials. Pantos et al. [32], at the annual European Society of Human Reproduction and Embryology conference held in 2016 in Helsinki, Finland, introduced this modality (ovarian rejuvenation). They injected PRP in eight perimenopausal/POF women with poor ovarian reserve. They found successful ovarian rejuvenation 1-3 months after PRP treatment. All cases underwent natural IVF cycles with follicles of $15.20 \pm 2.05 \mathrm{~mm}$ in diameter, the resulting oocytes were inseminated by intracytoplasmic sperm injection (ICSI), and all resulting embryos were cryopreserved.

\section{2) PRP in ovarian torsion}

In an animal model, 60 adult female rats were subjected to ischemia and bilateral adnexal torsion for 3 hours. Intraperitoneal PRP was administered 30 minutes prior to ischemia in one group, while the other group was not injected with PRP. Detorsion was then done, and oxidative stress levels, histopathological changes, and reperfu- 
sion injuries were lower in the PRP group than in the other group. The researchers concluded that PRP was effective for the prevention of ischemia and reperfusion damage in rat ovary [33].

\section{3) PRP in refractory endometrium}

The endometrium plays an important role in achieving optimal outcomes of assisted reproductive technologies. Endometrial growth following inadequate ovarian stimulation may be insufficient, leading to poor results of IVF/ICSI cycles. Various strategies been suggested to improve endometrial thickness, especially in resistant cases. PRP is a novel therapy that has been tried in such patients [34].

Colombo et al. [35] included eight patients who underwent PRP treatment. The inclusion criteria were women with more than three cancelled cryo-transfers due to poor endometrial growth ( $<6 \mathrm{~mm}$ ), women with a negative hysteroscopic screening for endometrial pathology, and women with a negative bacteriologic screening. After application of PRP, the endometrial thickness was satisfactory in seven cases. A positive test for beta-human chorionic gonadotropin was found in six women. They concluded that the multiple implantation failures were caused by inefficient expression of adhesion molecules, which could potentially be improved by PRP application.

Similarly, Zadehmodarres et al. [36], in their pilot study, included 10 patients who had a history of cancelled cycles due to inadequate endometrial growth $(<7 \mathrm{~mm})$. They found that endometrial thickness increased at 48 hours after the first PRP application and reached more than $7 \mathrm{~mm}$ after the second PRP application in all patients. Embryo transfer was then carried out in all patients. Five patients became pregnant (50\%), and in four of them the pregnancy progressed normally. They concluded that PRP was effective for endometrial growth in patients with a thin endometrium.

Jang et al. [37], in an animal model, investigated the role of PRP in the regeneration of the endometrium after ethanol-induced damage. They found that intrauterine administration of autologous PRP stimulated and accelerated regeneration of the endometrium, as well as decreasing fibrosis, in a murine model of endometrial damage.

\section{4) PRP in repeated implantation failure}

Repeated implantation failure (RIF) is defined as failure to conceive following several embryo transfers in IVF cycles. Numerous factors are involved in the implantation process, including embryo quality, endometrial receptivity, and immunological factors [38].

Several methods have been suggested for RIF management, but little consensus exists on which is most effective. These methods include blastocyst transfer, assisted hatching, hysteroscopy, endometrial scratching, and immune therapy. Recently, the intrauterine infusion of PRP has been described as a way to promote endometrial growth and receptivity [34,39].
Nazari et al. [40] enrolled 20 participants with a history of RIF to evaluate the effectiveness of PRP in improving the pregnancy rate in RIF patients. The inclusion criteria were being younger than 40 years and having a BMl below $30 \mathrm{~kg} / \mathrm{m}^{2}$. They reported that 18 of the 20 participants (90\%) became pregnant. Sixteen clinical pregnancies were recorded, and their pregnancies were ongoing at the time of the study. They concluded that PRP was effective in improving pregnancy outcomes in RIF patients.

\section{PRP in aesthetic gynecology \\ 1) $P R P$ in breast reconstruction}

Many studies of PRP have been conducted in the field of aesthetic and plastic surgery, but all were pilot studies, had small samples, or used animal models. PRP together with adipose tissue has been used in breast reconstruction [41]. Gentile et al. [42] enrolled 100 patients aged between 19 and 60 years affected by breast soft-tissue defects. They divided the patients into two equally-sized groups. The study group was treated with fat grafting and PRP, while the control group was treated with fat grafting injections only. They found that the patients treated with PRP added to the autologous fat grafts showed a $69 \%$ maintenance rate of the restored contour and of three-dimensional volume after 1 year, whereas the patients in the control group showed a 39\% maintenance rate. They concluded that PRP mixed with fat grafts led to improvements in the maintenance of breast volume in patients affected by breast soft-tissue defects. Similar results were obtained by Salgarello et al. [43].

\section{2) PRP in female sexual dysfunction}

Platelets have been found to release around 35 growth factors that promote tissue regrowth, healing, and regeneration. This fact has been utilized by aesthetic gynecologists in treatments such as vaginal rejuvenation and 0-shot therapy [44].

\section{(1) O-Shot therapy}

PRP use in sexual dysfunction is considered to be a revolutionary new nonsurgical outpatient treatment that helps improve both urinary incontinence and sexual dysfunction through using a woman's own growth factors. The PRP is injected into specific areas of the vagina with the aid of local anesthetic cream. This modality of treatment is called the "O-shot." PRP immediately activates tissue regeneration, and the enhancement in sexual response is dramatic. The desired response includes improved arousal, stronger orgasm, decreased dyspareunia, and increased natural lubrication [45].

Runels et al. [46] enrolled 11 women who presented with dyspareunia. They injected PRP into the clitoris and vagina, and found that intravaginal and intraclitoral PRP injections could be an effective method to treat certain types of female sexual dysfunction, especial- 
ly in the areas of desire, arousal, lubrication, and orgasm.

\section{(2) Vaginal rejuvenation}

Aesthetic practitioners have used PRP for the regeneration of vaginal mucosa, muscles, and skin. After PRP injection, vaginal vascularity is increased, with a subsequent dramatic increase in sensitivity. In addition, the skin becomes thicker and firmer, making the vagina look much more youthful. Moreover, the ligaments and muscles supporting the urethra become stronger, alleviating urinary incontinence [47].

Kim et al. [48] reported the use of PRP in a case of vaginal rejuvenation. They concluded that the application of autologous lipofilling mixed with PRP in a patient with vaginal atrophy produced relief of symptoms and contour restoration. The rejuvenated appearance of the external genitalia provided a pleasing cosmetic outcome to the patient.

\section{PRP in premature rupture of membranes}

Premature rupture of membranes occurs due to damage and tears in the fetal membrane, leading to congenital infections and poor neonatal outcomes. PRP was tried in an in vitro model to evaluate the ability of PRP to seal iatrogenic fetal membrane defects. This was done using single- and double-layer amnion models. The PRP plug was stable and attached firmly to the amnion tear. The authors concluded that they found experimental evidence that a PRP plug persisted for nearly 2 months in an amniotic fluid environment. It also provided waterproof sealing of iatrogenic defects in the amnion and chorion. Moreover, PRP stimulates cell growth and proliferation, and may thereby enhance the membrane-healing response [49].

\section{Conclusion}

PRP is an innovative therapeutic modality, as it is affordable, simple, easily performed, and effective. It is also a noninvasive modality with promising results and no side effects. In the field of gynecology, the few studies that have been conducted are pilot studies, case series, and case reports. The risks of PRP therapy as infection, bleeding, and nerve damage, appear to be minimal. Large randomized controlled studies are required to confirm its efficacy and safety in various gynecological disorders.

\section{Conflict of interest}

No potential conflict of interest relevant to this article was reported.

\section{References}

1. Dhillon RS, Schwarz EM, Maloney MD. Platelet-rich plasma ther- apy: future or trend? Arthritis Res Ther 2012;14:219.

2. Everts PA, Hoogbergen MM, Weber TA, Devilee RJ, van Monftort $\mathrm{G}$, de Hingh $\mathrm{IH}$. Is the use of autologous platelet-rich plasma gels in gynecologic, cardiac, and general, reconstructive surgery beneficial? Curr Pharm Biotechnol 2012;13:1163-72.

3. Lai LP, Stitik TP, Foye PM, Georgy JS, Patibanda V, Chen B. Use of platelet-rich plasma in intra-articular knee injections for osteoarthritis: a systematic review. PM R 2015;7:637-48.

4. Wang SZ, Chang Q, Lu J, Wang C. Growth factors and plateletrich plasma: promising biological strategies for early intervertebral disc degeneration. Int Orthop 2015;39:927-34.

5. Amable PR, Carias RB, Teixeira MV, da Cruz Pacheco I, Correa do Amaral RJ, Granjeiro JM, et al. Platelet-rich plasma preparation for regenerative medicine: optimization and quantification of cytokines and growth factors. Stem Cell Res Ther 2013;4:67.

6. Dhurat R, Sukesh M. Principles and methods of preparation of platelet-rich plasma: a review and author's perspective. J Cutan Aesthet Surg 2014;7:189-97.

7. Anitua E, Sanchez M, Orive G. The importance of understanding what is platelet-rich growth factor (PRGF) and what is not. J Shoulder Elbow Surg 2011;20:e23-4.

8. Dohan Ehrenfest DM, Andia I, Zumstein MA, Zhang CQ, Pinto NR, Bielecki T. Classification of platelet concentrates (platelet-rich plasma-PRP, platelet-rich fibrin-PRF) for topical and infiltrative use in orthopedic and sports medicine: current consensus, clinical implications and perspectives. Muscles Ligaments Tendons J 2014;4:3-9.

9. Dohan Ehrenfest DM, Bielecki T, Mishra A, Borzini P, Inchingolo F, Sammartino $G$, et al. In search of a consensus terminology in the field of platelet concentrates for surgical use: platelet-rich plasma (PRP), platelet-rich fibrin (PRF), fibrin gel polymerization and leukocytes. Curr Pharm Biotechnol 2012;13:1131-7.

10. Mishra A, Harmon K, Woodall J, Vieira A. Sports medicine applications of platelet rich plasma. Curr Pharm Biotechnol 2012;13:118595.

11. Magalon J, Chateau AL, Bertrand B, Louis ML, Silvestre A, Giraudo $L$, et al. DEPA classification: a proposal for standardising PRP use and a retrospective application of available devices. BMJ Open Sport Exerc Med 2016;2:e000060.

12. Jo CH, Roh YH, Kim JE, Shin S, Yoon KS. Optimizing platelet-rich plasma gel formation by varying time and gravitational forces during centrifugation. J Oral Implantol 2013;39:525-32.

13. International Cellular Medicine Society. Platelet Rich plasma (PRP) guidelines [Internet]. Las Vegas: International Cellular Medicine Society; 2011 [cited 2018 Jun 18]. Available from: http://www.cellmedicinesociety.org/icms-guidelines/guidelines. 14. Sundman EA, Cole BJ, Karas V, Della Valle C, Tetreault MW, Mo- 
hammed $\mathrm{HO}$, et al. The anti-inflammatory and matrix restorative mechanisms of platelet-rich plasma in osteoarthritis. Am J Sports Med 2014;42:35-41.

15. Sunitha Raja V, Munirathnam Naidu E. Platelet-rich fibrin: evolution of a second-generation platelet concentrate. Indian J Dent Res 2008;19:42-6.

16. Tehranian A, Esfehani-Mehr B, Pirjani R, Rezaei N, Sadat Heidary S, Sepidarkish M. Application of autologous platelet-rich plasma (PRP) on wound healing after caesarean section in high-risk patients. Iran Red Crescent Med J 2016;18:e34449.

17. Fanning J, Murrain L, Flora R, Hutchings T, Johnson JM, Fenton BW. Phase I/II prospective trial of autologous platelet tissue graft in gynecologic surgery. J Minim Invasive Gynecol 2007;14:633-7.

18. Hua X, Zeng Y, Zhang R, Wang H, Diao J, Zhang P. Using plateletrich plasma for the treatment of symptomatic cervical ectopy. Int J Gynaecol Obstet 2012;119:26-9.

19. Casabona F, Priano V, Vallerino V, Cogliandro A, Lavagnino G. New surgical approach to lichen sclerosus of the vulva: the role of adipose-derived mesenchymal cells and platelet-rich plasma in tissue regeneration. Plast Reconstr Surg 2010;126:210e-211e.

20. Behnia-Willison F, Pour NR, Mohamadi B, Willison N, Rock M, Holten IW, et al. Use of platelet-rich plasma for vulvovaginal autoimmune conditions like lichen sclerosus. Plast Reconstr Surg Glob Open 2016;4:e1124.

21. Morelli M, Rocca ML, Venturella R, Di Cello A, Del Negro S, Condorelli $M$, et al. Adjuvant use of platelet gel for wound breakdown prevention in advanced vulvar cancer surgery: a retrospective study. Int J Gynecol Cancer 2013;23:1490-4.

22. Bodner-Adler B, Hanzal E, Pablik E, Koelbl H, Bodner K. Management of vesicovaginal fistulas (VVFs) in women following benign gynaecologic surgery: a systematic review and meta-analysis. PLoS One 2017;12:e0171554.

23. Shirvan MK, Alamdari DH, Ghoreifi A. A novel method for iatrogenic vesicovaginal fistula treatment: autologous platelet rich plasma injection and platelet rich fibrin glue interposition. J Urol 2013;189:2125-9.

24. Mongardini M, lachetta RP, Cola A, Maturo A, Giofre M, Custureri F. Low rectovaginal fistula treated with platelet-rich plasma (PRP). G Chir 2009;30:507-9.

25. Gottgens KW, Smeets RR, Stassen LP, Beets GL, Pierik M, Breukink SO. Treatment of Crohn's disease-related high perianal fistulas combining the mucosa advancement flap with platelet-rich plasma: a pilot study. Tech Coloproctol 2015;19:455-9.

26. Gorlero F, Glorio M, Lorenzi P, Bruno-Franco M, Mazzei C. New approach in vaginal prolapse repair: mini-invasive surgery associated with application of platelet-rich fibrin. Int Urogynecol J 2012;23:715-22.
27. Medel S, Alarab M, Kufaishi H, Drutz H, Shynlova O. Attachment of primary vaginal fibroblasts to absorbable and nonabsorbable implant materials coated with platelet-rich plasma: potential application in pelvic organ prolapse surgery. Female Pelvic Med Reconstr Surg 2015;21:190-7.

28. Chrysanthopoulou EL, Pergialiotis V, Perrea D, Kourkoulis S, Verikokos C, Doumouchtsis SK. Platelet rich plasma as a minimally invasive approach to uterine prolapse. Med Hypotheses 2017; 104:97-100.

29. Nikolopoulos KI, Pergialiotis V, Perrea D, Doumouchtsis SK. Restoration of the pubourethral ligament with platelet rich plasma for the treatment of stress urinary incontinence. Med Hypotheses 2016;90:29-31.

30. Einarsson Jl, Jonsdottir K, Mandle R. Use of autologous platelet gel in female pelvic organ prolapse surgery: a feasibility study. J Minim Invasive Gynecol 2009;16:204-7.

31. White YA, Woods DC, Takai Y, Ishihara O, Seki H, Tilly JL. Oocyte formation by mitotically active germ cells purified from ovaries of reproductive-age women. Nat Med 2012;18:413-21.

32. Pantos K, Nitsos N, Kokkali G, Vaxevanoglou T, Markomichali C, Pantou A, et al. Ovarian rejuvenation and folliculogenesis reactivation in peri-menopausal women after autologous plateletrich plasma treatment. Proceedings of the 32nd Annual Meeting of ESHRE; 2016 July 3-6; Helsinki, Finland.

33. Bakacak M, Bostanci MS, Inanc F, Yaylali A, Serin S, Attar R, et al. Protective effect of platelet rich plasma on experimental ischemia/ reperfusion injury in rat ovary. Gynecol Obstet Invest 2016;81:22531.

34. Garcia-Velasco JA, Acevedo B, Alvarez C, Alvarez M, Bellver J, Fontes J, et al. Strategies to manage refractory endometrium: state of the art in 2016. Reprod Biomed Online 2016;32:474-89.

35. Colombo GV, Fanton V, Sosa D, Criado Scholz E, Lotti J, Aragona $\mathrm{SE}$, et al. Use of platelet rich plasma in human infertility. J Biol Regul Homeost Agents 2017;31(2 Suppl. 2):179-82.

36. Zadehmodarres S, Salehpour S, Saharkhiz N, Nazari L. Treatment of thin endometrium with autologous platelet-rich plasma: a pilot study. JBRA Assist Reprod 2017;21:54-6.

37. Jang HY, Myoung SM, Choe JM, Kim T, Cheon YP, Kim YM, et al. Effects of autologous platelet-rich plasma on regeneration of damaged endometrium in female rats. Yonsei Med J 2017;58:1195-203.

38. Salehpour S, Zamaniyan M, Saharkhiz N, Zadeh Modares S, Hosieni $S$, Seif $S$, et al. Does intrauterine saline infusion by intrauterine insemination (IUI) catheter as endometrial injury during IVF cycles improve pregnancy outcomes among patients with recurrent implantation failure? An RCT. Int J Reprod Biomed (Yazd) 2016;14:583-8.

39. Chang Y, Li J, Chen Y, Wei L, Yang X, Shi Y, et al. Autologous plate- 
let-rich plasma promotes endometrial growth and improves pregnancy outcome during in vitro fertilization. Int J Clin Exp Med 2015;8:1286-90.

40. Nazari L, Salehpour S, Hoseini S, Zadehmodarres S, Ajori L. Effects of autologous platelet-rich plasma on implantation and pregnancy in repeated implantation failure: a pilot study. Int J Reprod Biomed (Yazd) 2016;14:625-8.

41. Jin R, Zhang L, Zhang YG. Does platelet-rich plasma enhance the survival of grafted fat? An update review. Int J Clin Exp Med 2013;6:252-8.

42. Gentile P, Di Pasquali C, Bocchini I, Floris M, Eleonora T, Fiaschetti $V$, et al. Breast reconstruction with autologous fat graft mixed with platelet-rich plasma. Surg Innov 2013;20:370-6.

43. Salgarello M, Visconti G, Rusciani A. Breast fat grafting with platelet-rich plasma: a comparative clinical study and current state of the art. Plast Reconstr Surg 2011;127:2176-85.

44. Ramos-E-Silva M, Fonteles LA, Lagalhard CS, Fucci-da-Costa AP. Stylage $\AA$ : a range of hyaluronic acid dermal fillers containing mannitol: physical properties and review of the literature. Clin Cosmet Investig Dermatol 2013;6:257-61.
45. Jain A, Bedi RK, Mittal K. Platelet-rich plasma therapy: a novel application in regenerative medicine. Asian J Transfus Sci 2015;9: 113-4.

46. Runels C, Melnick H, Debourbon E, Roy L. A pilot study of the effect of localized injections of autologous platelet rich plasma (PRP) for the treatment of female sexual dysfunction. J Womens Health Care 2014;3:169.

47. Ruth Styles. Would you plump up your vagina with fillers? Top cosmetic doctor warns of risky new trend after being inundated with clients looking for 'genital enhancements' [Internet]. London: Daily Mail; 2015 [cited 2018 Jun 17]. Available from: http:// www.dailymail.co.uk/femail/article-3114937/Top-cosmeticdoctor-warns-risky-new-trend-genital-filler-jabs.html.

48. Kim SH, Park ES, Kim TH. Rejuvenation using platelet-rich plasma and lipofilling for vaginal atrophy and lichen sclerosus. J Menopausal Med 2017;23:63-8.

49. Lewi L, Liekens D, Heyns L, Poliard E, Beutels E, Deprest J, et al. In vitro evaluation of the ability of platelet-rich plasma to seal an iatrogenic fetal membrane defect. Prenat Diagn 2009;29:620-5. 